\title{
Spatial Regions Periodicity based Detection of Two-wheelers using Histogram of Oriented Gradients
}

\author{
Yeunghak Lee ${ }^{1}$, Taesun $\mathrm{Kim}^{2}$, Sanghoon Lee ${ }^{3}$ and Jaechang Shim ${ }^{4}$ \\ Kyungwoon University ${ }^{1,2}$ \\ $G E R I^{3}$ \\ Andong National University ${ }^{4}$ \\ annaturu@ikw.ac.kr,tskim@ukw.ac.kr,legotec@geri.re.kr,jcshim@andong.ac.kr
}

\begin{abstract}
Recent research has been devoted and focused on detecting pedestrians and vehicles in intelligent vehicles except for vulnerable road users (VRUs). Histogram of Oriented Gradients (HOGs) descriptor is one of the state-of-art methods which have been shown to significantly outperform the existing feature set for several objects detection. In this paper we present a method for two-wheelers detection based on the periodicity of the size of spatial regions; cells and blocks. Contrary to concatenating feature vectors using a dense grid of HOG, proposed algorithm storage extracted feature vectors according to the period in each step. Experimental results show that the new approach gives higher detection accuracy than that of the conventional method.
\end{abstract}

Keywords: HOG; two-wheelers; local vectors; spatial regions; Adaboost

\section{Introduction}

Not only vehicles and people, but also various types of transportation are running on the country and city roads. But while a simple device or vehicle to move things may be in past, the shape (or style) and function has quickly and variously progressed until now. The development of vehicles has been concentrated on not only improving performance, but also to protect the drivers and passengers in a vehicle in the occurrence of a traffic accidents. Especially airbag, bumper, electronic equipments, and etc., has been focused on protecting drivers from accident on the road and enhancing comfortability until a few years ago. But the accidents outside of a vehicle, caused by a driver's carelessness or by road environments, still have many problems, even though traffic accidents has been reduced gradually by safety devices, so it is required to detect the risk of a traffic accident in order to save lives [1].

Most of the studies relating to human safety over the past several years have mainly concentrated on increasing the detection rate of pedestrians and automobiles on a road from a still image and framework. Lately, the study scope has expanded to protect vulnerable road users (VRUs), such as those consisting of humans, two-wheelers, small moving devices and other small vehicles [1-2]. Unlike pedestrian and vehicle recognition for intelligent vehicles, two-wheelers recognition appears as a combination of human and complicated objects. Because pedestrians and two-wheeler riders are very vulnerable road participants among VRUs, they are a hot subject for the study field in intelligent transportation system.

Various type of sensors are utilized for an accurate and real time detection and tracking, including near-infrared camera and far-infrared camera [3-5], which are very expensive and capable of operating even at night, LIDAR or RADAR [6], which is operated with a high frequency band, Laser Scanner [7], which is capable of obtaining and detecting a high amount of data in real time, and radio-based mobile communication [8] such as Global System for Universal Mobile Telecommunication(UMST), which are 
widely utilized even now due to the merits of those vision-based systems in spite of their attractiveness, pursuant to good expansion capability, low price, and low calculation $[9,10]$. Sometimes a fusion system is utilized. And this is still a challenging problem because of the fact that people and two-wheelers can appear to have quite different shape due to differences in the clothing/hairstyle, body pose, and two-wheelers model type, especially according to the view angles [11].

The automobile vision-based systems have mainly concentrated on recognizing pedestrians and automobiles [8]. Pedestrian detection has many algorithms that are similar to two wheelers detection. Because of this reason, our paper describes the pedestrian detection. Extracting characteristics for detecting pedestrians are classified with single characteristics, multiple characteristics, whole area characteristics, and district characteristics pursuant to the usage of extracted characteristics [12-14]. The whole area characteristics utilize the characteristics extracted with the whole image as the principal component analysis (PCA) does [15, 16]. On the contrary, the district characteristics extract characteristics from a partial area of the image and then extract the final characteristics through a sliding window. The single characteristics, widely used to detect pedestrians and objects, are incorporated edge [17], appearance [18], local binary pattern (LBP) [13, 19], histogram of oriented gradients (HOG) [20], Haar-like [21] and wavelet coefficient [22]. On the contrary, the multiple characteristics combine several single characteristics for making new characteristics and then utilize them [23]. The other methods improve detection rate by utilizing the characteristics during classification, including Cascade method [24], Support vector machine (SVM) [25], Neural network, and k-NN classifier [26]. HOG characteristics or improved HOG characteristics are widely utilized in the methods for recognizing pedestrians by using the automobile vision. Zhu et al. [27] applied the HOG characteristics based on variable block size to improve detection speed. Further, Watanabe et al. [28] utilized co-occurrence HOG characteristics, and Wang et al. [23] utilized HOG-LBP human detection to improve detection accuracy.

The motivation of this paper is as follows. Firstly, two wheelers detection system does not have a considerable time investment to find a good algorithm. Secondly, it is familiar with pedestrian detection which has accuracy and efficiency in still images. But it is one of the most difficult works due to a variety range of poses, as well as environmental conditions, cluttered backgrounds, and composite object (shown variety shapes than pedestrian, according to the view point). So in this paper, we suggest a new algorithm to detect the two-wheelers using subimage periodicity for the weak part on the road.

This paper is organized as follow. In Section 2, this paper explains basic extracting characteristics methods with HOG and a dramatic algorithm that can increase detection rates significantly. Section 3 descries Adaboost algorithm used in training and classification of suggested detecting two-wheelers system. The evaluation and detailed analysis for the experimental results are summarized in Section 4. Section 5 concludes this paper.

\section{HOG Feature Description}

HOG [20] converts the distribution directions of brightness for a local region into a histogram to express them in feature vectors, which is utilized to express the shape characteristics of an object. And it is influenced a little from an effect of illumination by converting the distribution of near pixels for a local region into a histogram, and has a strong feature for a geometric change of local regions. The following is a detailed explanation on how HOG description is calculated. 


\subsection{Gradient Computation}

Value of gradient at every image pixel $I(x, y)$ is calculated by derivatives $f_{\mathrm{x}}$ and $f_{\mathrm{y}}$ in $\mathrm{x}$ and y direction by convolving the filter mask $\left[\begin{array}{lll}-1 & 0 & 1\end{array}\right]$ and $\left[\begin{array}{lll}-1 & 0 & 1\end{array}\right]^{\mathrm{T}}$. Refer equation (1) and (2).

$$
\begin{aligned}
& f_{x}=I \otimes\left[\begin{array}{lll}
-1 & 0 & 1
\end{array}\right] \\
& f_{y}=I \otimes\left[\begin{array}{lll}
-1 & 0 & 1
\end{array}\right]^{T}
\end{aligned}
$$

where $I$ is a example gray scale image and $\otimes$ is the convolution operation.

The gradient magnitudes $m(x, y)$ and orientation direction $\theta(x, y)$ for each pixel are calculated by

\subsection{Orientation Binning}

$$
\begin{gathered}
m(x, y)=\sqrt{f_{x}^{2}+f_{y}^{2}} \\
\theta(x, y)=\arctan \left(\frac{f_{y}}{f_{x}}\right)
\end{gathered}
$$

This stage defines production of an encoding that is sensitive to local image content. The image windows are divided into $8 \times 8$ rectangular small spatial regions call cells, as shown in Figure 1 (c). Similar to [20], we used unsigned gradients in conjunction with nine bins for every cell (a bin corresponds to $20^{\circ}$ ). The $8 \times 8$ cell magnitude pixels are accumulated in one of the nine bins according to their orientation direction. Figure 1 (c) depicts a graphical representation on how the gradient angle range is binned in its respective cell.

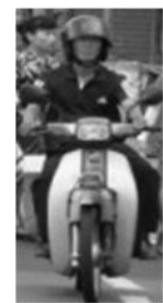

(a)

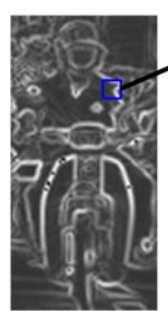

(b)

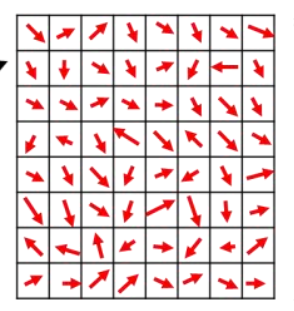

(c)

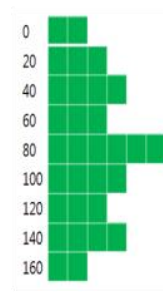

(d)

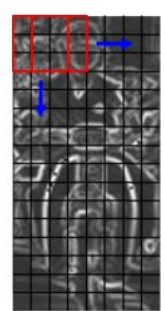

Figure 1. The Example of Two Wheelers HOG Normalization; (a) Original Image (b) Calculated Magnitude vector (c) Cell and Orientation Binning Expression (d) Normalization by Blocks for $3 \times 3$ Cells

\subsection{Block Normalization}

Directional histograms for brightness prepared in each of the cells were normalized as a block of $3 \times 3$ cells. This is performed by grouping cells in larger spatial regions called blocks. Characteristic quantities (9 dimensions) of row i, column $\mathrm{j}$, Cell $(\mathrm{i}, \mathrm{j})$ is expressed as $\mathrm{F}_{\mathrm{i}, \mathrm{i}}=\left[\mathrm{F}_{1}, \mathrm{~F}_{1}, \cdots, \mathrm{F}_{9}\right]$. The characteristic quantities of $k$ 'th block (81 dimensions) may be expressed as:

$$
B_{k}=\left\lfloor F_{i j}, F_{i+1 j}, F_{i+2 j}, F_{i j+1}, F_{i j+2}, F_{i+1 j+1}, F_{i+2 j+1}, F_{i+1 j+2}, F_{i+2 j+2}\right\rfloor
$$

Normalization processes are summarized in figure 1 (d), where a movement of block is based on fact is moved to the right side and to the lower side by one cell 
each. The overlapping process is done to ensure the important features of each cell. The normalized characteristic vectors for each block are given by

$$
\Pi=\frac{B_{k}}{\sqrt{\left\|B_{k}\right\|^{2}+\varepsilon^{2}}}(\varepsilon=1)
$$

And the feature vectors are saved by concatenation method. For example, the dimension number for the height and width of an input image are 128x64 pixels, the dimension number of the histogram is 9 , the size of cell is 8 , and the size of block is 3 , then the calculated the number of HOG feature vectors with 6804 dimension is obtained.

\subsection{Proposed HOG Features}

Out proposed HOG descriptors are based on dense and overlapping encoding of small spatial image regions (cells) and large spatial image regions (blocks). First, each cell contains orientation bins. In general, cell characteristic values obtained from each cell are expressed by concatenation method, as multi dimension cell space, which are equal to Eq. (7) and Figure 2 (a):

$$
\Gamma=\left[\begin{array}{llll}
C_{1} & C_{2} & \ldots & C_{n}
\end{array}\right]
$$

Proposed cell histogram features are saved at an interval of cell number as shown in Figure 2 (b), unlike the general method. For example, if each cell has $8 \times 8$ pixels for original image (128x64), the number of cell is 128 . And the features are saved by 128 periods. The method suggested in this study to save feature vectors is to save feature vectors using the thinned out method for each cell, according to cell numbers, as shown Figure 2 (b).

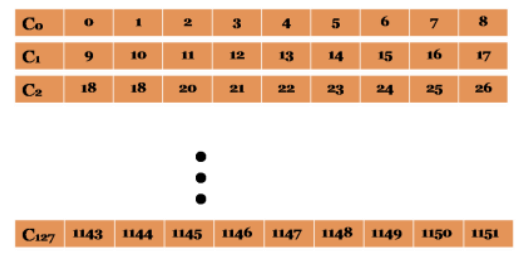

(a)

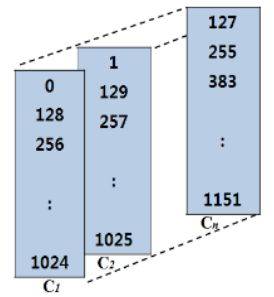

(b)

\section{Figure 2. Cell Feature Vectors (a) Concatenation Method (b) Proposed Method Using Periodicity of Total Cell Size}

Second, the block grid ( $\mathrm{n} \times \mathrm{n}$ ) which define the number of cells in each block is large spatial regions having overlapping. Generally block features are saved by concatenation method, as shown in Figure 3 (a). Before the block normalization, each block transposes to scatter the features according to the rule, as shown Figure 3 (b). Similar to the cells, we calculate the number of blocks. Then proposed block features are saved by the block period, as shown Figure 3 (c). 


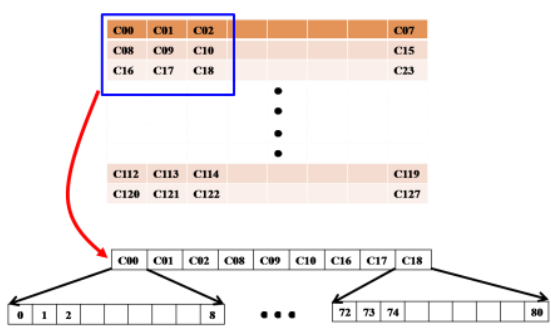

(a)

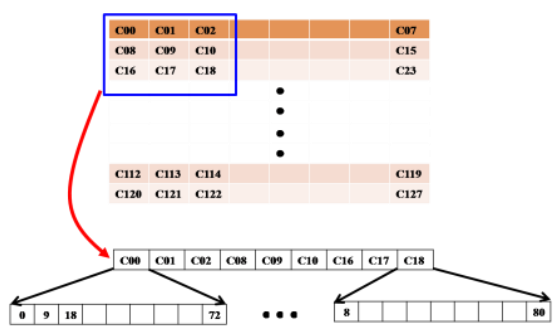

(b)

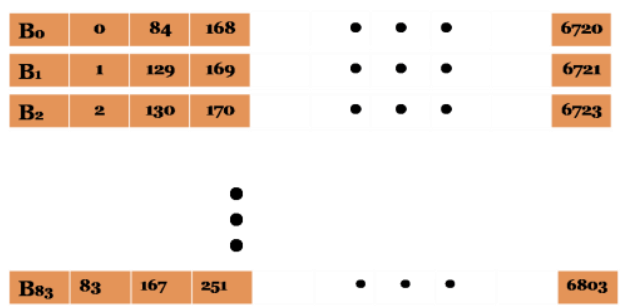

(c)

Figure 3. Block Feature Vectors (a) the Location of General Block Features (b) Transpose of Block Features (c) Proposed Block Features using Block Periodicity

Characteristic vectors are saved in the position information within each of the cells and blocks. The images used in this study are expressed by utilizing the characteristic vector saving positions and the matrix positions that each of the cells has, of which are shown in Figure 3 (c). Therefore, each of the cells and blocks has a scattering characteristic vector at positions with same interval for dense and overlapping processing, which is placed through whole cells and blocks.

\section{Classification}

SVM Method and Adaboost Method are most widely utilized for classifying objects. SVM Method requires many calculations (or memory space) and a lot of discrimination time. On the contrary, Adaboost method [29] has merits of speedy classification and no additional factors other than the round of a characteristic vector. Therefore, in this study, an Adaboost algorithm was utilized to detect twowheelers by using the characteristic vectors of the two-wheelers for the input images that were conducted earlier. The processes are summarized as below.

I. Input: two-wheelers $(+1)$ and non two-wheelers $(-1)$

Set of training images: $\left(x_{1}, y_{1}\right), \cdots,\left(x_{n}, y_{n}\right)$ where $x_{i} \in X, \quad y_{i} \in Y=\{+1,-1\}$

II. Initialize weighting factors.

$w_{1, i}=1 / 2 m, 1 / 2 l$ for $y_{i}=+1,-1$

$m$ : the number of positive image (two-wheeler, +1 )

$l$ : the number of negative image (non two-wheeler, -1)

III. For $t=1 \ldots T$

(a) Normalize the weights,

$$
w_{t, i}=\frac{w_{t, i}}{\sum_{j=1}^{n} w_{t, j}}
$$

$w_{t, i}$ is a probability distribution of $i$ th training image for th weak classification. 
(b) For each feature, $\mathrm{j}$, training a classifier $h_{j}$ which is restricted to using a single feature. The error is evaluated with respect to $w_{i}$

$$
\varepsilon_{j}=\sum_{i} w_{j}\left|h_{j}\left(x_{i}\right)-y_{i}\right|
$$

(c) Choose the classifier, $h_{t}$, with the lowest error $\varepsilon_{t}$

(d) Update the weights: $w_{t+1, i}=w_{t, i} \beta_{t}^{1-\varepsilon_{i}}$

where $\varepsilon_{i}=-1$ if example $x_{i}$ is classified correctly, $\varepsilon_{i}=+1$ otherwise, and

$$
\beta_{t}^{i}=\frac{\varepsilon_{t}}{1-\varepsilon_{t}}
$$

IV. Finally, the strong classifier becomes,

$$
H(x)=\operatorname{sign}\left(\sum_{t=1}^{T} \alpha_{t} h_{t}(x)\right) \text { Where } \alpha_{t}=\log \left(1 / \beta_{t}\right)
$$

In Stage I, initial input value $\mathrm{x}$ represents the training image, and y represents classification of positive (+1) and negative (-1). Stage II conducts initial weighting factors. Stage III creates weak classifiers, in which it selects a weak classifier having a minimum error and imposes a weighting factor to the classifier.

\section{Experimental Results}

In this study, an experiment was carried out with an ordinary user computer environment consisting of a Pentium 3.1 GHz and Visual C++ 6.0 Program. Since there is no public dataset for two-wheeler images, we built such a dataset by ourselves, using a digital camera and websites. An image of two-wheelers can be expressed by various angles in an automobile. For our purposes, it is hypothesized in the experiment for the following 2 cases: a two-wheeler is running in front of an automobile (rear appearance) and a two-wheeler is coming toward the automobile (front appearance). The experiment was done for an attitude of 90 degrees and an attitude of within 60 degrees in basis of a horizontal line. 2,353 pictures of normalized two-wheelers were used sized at 128x64 from the original photos sized at $640 \times 480$, which were utilized by dividing the training image and the experimental image. Pictures of non two-wheelers were obtained by utilizing randomly extracted pictures from the photos of streets in ordinary cities. The number of non two-wheelers used in the training was equal to the number of twowheelers, and 3,000 pictures of non two-wheelers were used in the experiment. An example of the images used in the experiment is shown in figure. 4. In figure 4, the first row refers to the images of two-wheelers corresponding to bicycles and the second row refers to the images corresponding to motorcycles.

The experiment was carried out using ordinary HOG and the spatial region periodicity method (P_HOG), which is suggested in the study; then a Haar-like experiment was conducted by applying the method. A range of thresholds of -20 to 20 was utilized in classification, and confusion matrix, true positive rate (TPR) and false positive rate (FPR) were used for analyzing experimental results per angles for the methods, and ROC curves are shown in figure. 5, by applying Eq. (8) below:

$$
T P R=\frac{T P}{T P+F N}, F P R=\frac{F P}{F P+T N}
$$

where "TP" is True Positive", "FP" is False Positive", "TN" is True Negative and "FN" is False Negative. In figure 3, "Moto" means motorcycle, "Bike" means bicycle, and "MB" is a mixture of motorcycles and bicycles, respectively. Also, the numerals behind each of 
the abbreviations " 60 " signifies within 60 degrees, " 90 " within 90 degrees, and "90-60" a mixture of 90 and 60 degrees, respectively, as well.

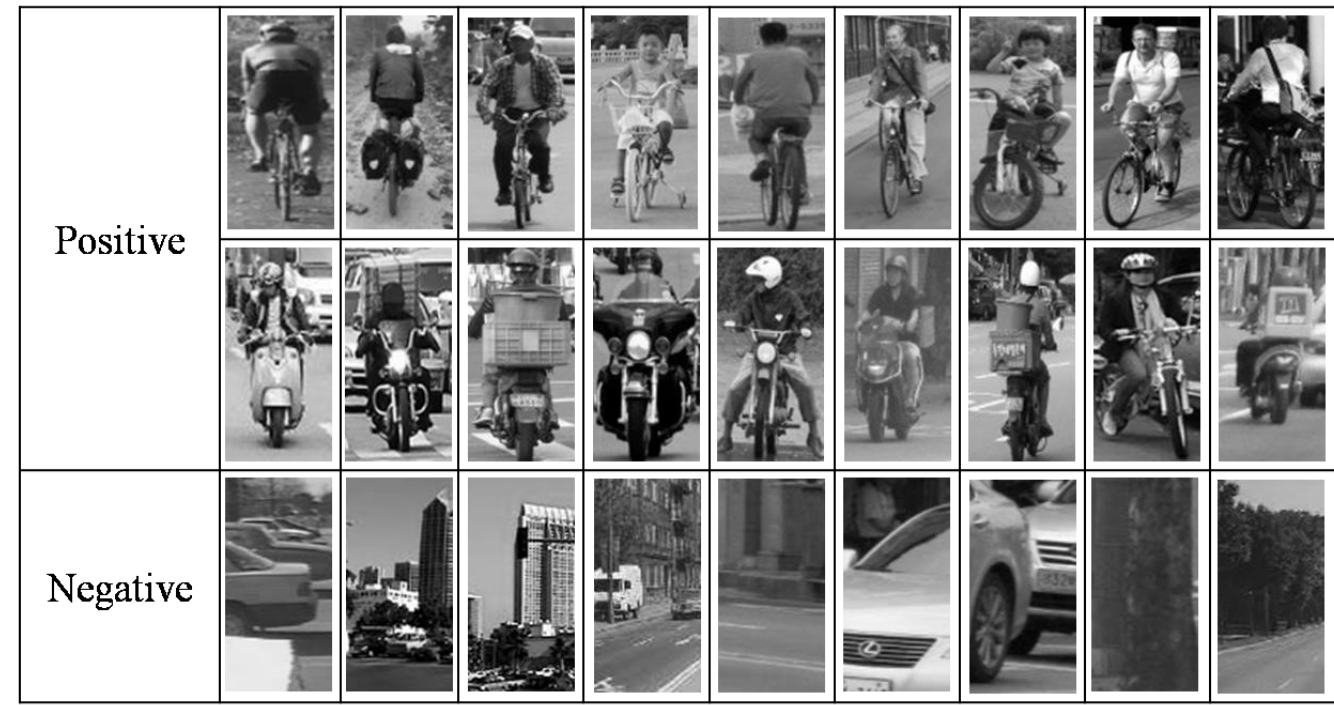

Figure 4. Example of Database Set for Positive and Negative

In Figure 5 (a), it has shown that the experiment according to "MB" has the best results among these experiments, but the recognition rate is significantly low. However, in Figure 5 (b), shows that the results of MB experiments according to the suggested method have a recognition rate of nearly 1 . When applied with another analytical method, as shown in Figure 5 (b) and (c), because the area of the curve of the suggested method is larger than that of the curve of the ordinary method, it is known that this system has a better performance. When P_HOG algorithm was applied for the other characteristic vector (Haar-like) method, a higher recognition rate could be obtained, and the results are listed in Figure 5 (c).

The highest accuracies for each of the methods were calculated with Eq. (9) [35] and the results are listed in Table 1. In Equation 9, True Positive signifies to discrimination of an image of two-wheeler for two-wheeler, but False Positive means to recognize an image of two-wheeler for non two-wheeler. In Table 1, "90-60" means the experiment with a mixture of 90 degrees and 60 degrees.

$$
\text { Accuracy }=\frac{T P}{T P+F P}
$$

As shown in Table 1, Bike (bicycle) has higher accuracies than Moto (motorcycle), signifying that bicycle has a trend of better classifying characteristics than motorcycle, in our opinion because a motorcycle becomes complicated by loading baggage at the rear or by the high loading of baggage. In the experiment of mixture of the two kinds of twowheelers, the results of accuracies have partially confused patterns for angles and shapes, but for the whole mixture (90-60) the suggested method has a higher accuracy than the existing algorithm. 


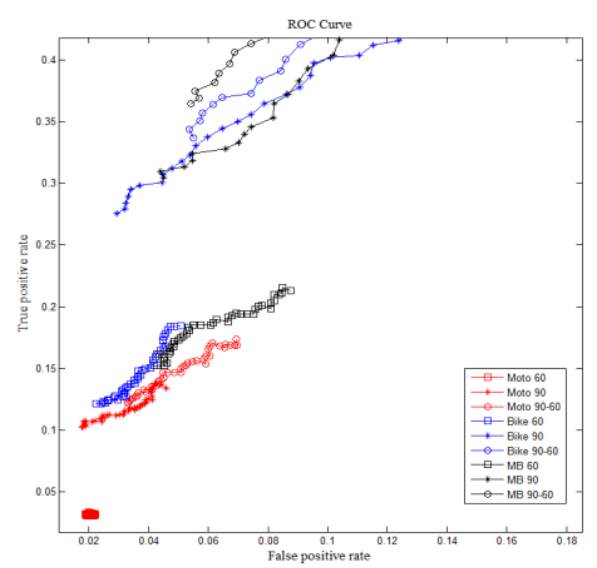

(a)

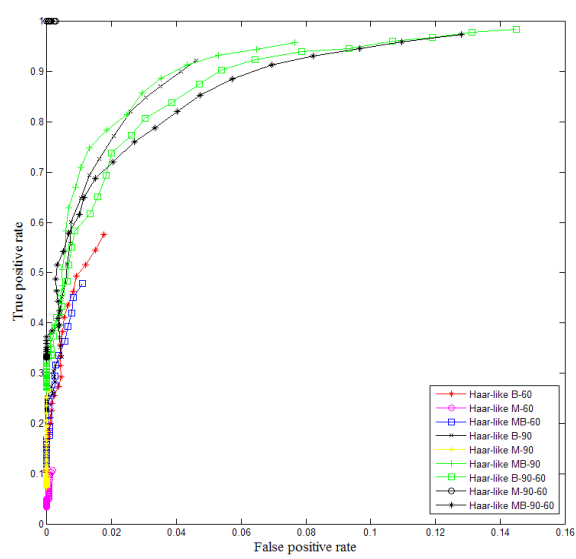

(b)

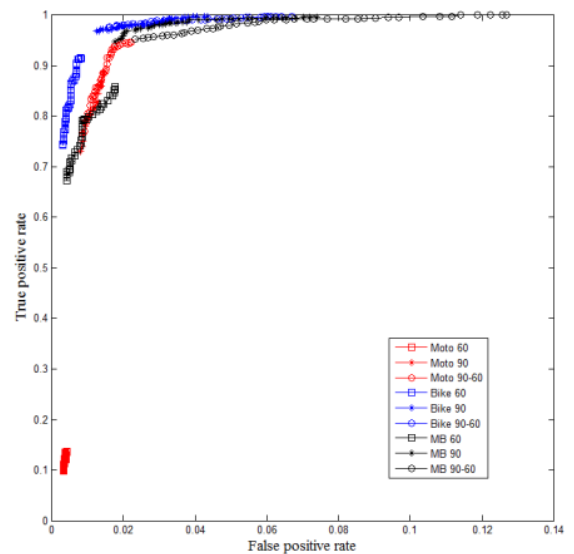

(c)

Figure 5. Experiment Results (a) Results of an ordinary HOG Method,

(b) Results of the Haar-like Experiment by Applying the Suggested Method, (c) Results of the HOG Experiment by Applying the Suggested Algorithm

Table 1. Accuracies for Each of the Methods

\begin{tabular}{|l|c|c|c|c|c|c|c|c|c|}
\hline \multirow{2}{*}{ Method Angle } & \multicolumn{3}{|c|}{60} & \multicolumn{3}{c|}{90} & \multicolumn{3}{c|}{$90-60$} \\
\cline { 2 - 10 } & M & B & MB & M & B & MB & M & B & MB \\
\hline Haar-like & 79.3 & 92.8 & 87.7 & 81.3 & 94.8 & 94.6 & 98 & 93.5 & 92.5 \\
\hline HOG & 61.1 & 71.2 & 76.7 & 74.9 & 78.3 & 76.1 & 77.8 & 75.5 & 73.1 \\
\hline P_HOG & 83.2 & 97.5 & 96.8 & 97.5 & 98.2 & 97.2 & 97.5 & 97.8 & 97.0 \\
\hline
\end{tabular}

To improve the suggested algorithm using spatial region periodicity, we need to supplement this paper experiment for the local binary pattern (LBP), which is the most popular texture classification feature. Also it is robust to illumination and contrast variations as it only considers the signs of pixel differences. Additional experiments show that proposed algorithm achieves higher accuracy than the original algorithm 
using concatenation method, as shown in Table 2. Here, P_LBP means that the proposed algorithm is applied.

Table 2. Additional Experiment for Proposed Algorithm

\begin{tabular}{|l|c|c|c|c|c|c|c|c|c|}
\hline \multirow{2}{*}{ Method Angle } & \multicolumn{3}{c|}{60} & \multicolumn{3}{c|}{90} & \multicolumn{3}{c|}{$90-60$} \\
\cline { 2 - 12 } & M & B & MB & M & B & MB & M & B & MB \\
\hline LBP & 53.7 & 72.2 & 71.1 & 48.0 & 77.8 & 74.5 & 71.8 & 73.4 & 72.0 \\
\hline P_LBP & 63.1 & 92.3 & 92.4 & 87.4 & 95.7 & 93.6 & 90.8 & 94.7 & 92.9 \\
\hline HOG+LBP & 52.2 & 86.6 & 81.3 & 67.2 & 79.5 & 77.3 & 83.6 & 76.5 & 76.6 \\
\hline P_HOG+LBP & 76.8 & 94.0 & 94.4 & 95.4 & 97.3 & 96.2 & 94.5 & 96.7 & 95.6 \\
\hline
\end{tabular}

When the HOG of the ordinary method and the suggested method were applied, quite significant differences are shown in the ROC curve, which is similar to the Haar-like method applied with the suggested method, also; P_HOG method applied with the suggested algorithm has a higher performance. The results of detecting bicycle experiment applied with a threshold 10.0 according to Eq. (10) in actual 640x480image are shown in Figure. 6.

$$
B(t)= \begin{cases}1 & \sum_{t=1}^{T} h_{t}(x) H(x) \geq T h \\ 0 & \text { Otherwise }\end{cases}
$$

where $h_{t}(x)$ is $t$ 'th $128 \times 64$ image, $H(x)$ is strong classifier, $x$ is the characteristics vector and in the results of Eq. 10, "1" of $B(t)$ is to detect twowheelers and " 0 " is to detect non two-wheelers, respectively.

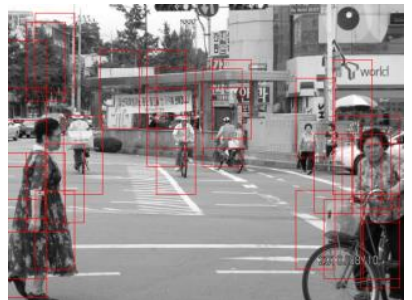

(a)

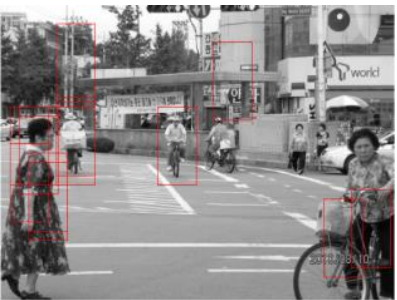

(b)

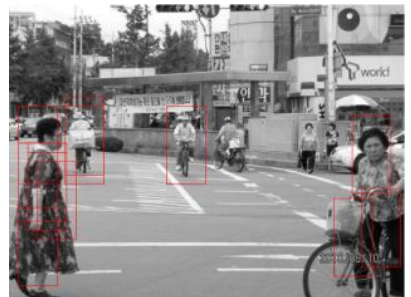

(c)

Figure 6. Example of the Results of $640 \times 480$ Application, (threshold value 10.0) (a) Haar-like Method, (b) HOG Method, (c) P_HOG Method (Proposed Method)

In Figure 7, for 640x480 images, a training image ratio of 1:1 and a mixture of 60 degrees and 90 degrees are applied. In the Figure, (a) shows the results of the ordinary HOG Method, (b) shows the results of the Haar-like Method, and c) shows the results of the T-HOG Method applied with the suggested method, respectively, with results showing that application of the suggested algorithm has much superior results than the existing methods. As demonstrated in the results, when a tree, electric pole, or street lamp were in close proximity with a person, it was detected as a two-wheeler; such problems will be supplemented in a future study. 
The results of the experiment per angle showed that the MB result of 90 degree have a higher system performance than 60 degrees, and the results of the mixture (noted as 90-60 in the Figure) has a average performance level of the two angles. Reasons for these results may be analyzed by the following two ideas: first, the number of trainings within 60 degrees was lower than 90 degrees, and second, since the characteristics of narrower ranges, which were almost similar to pedestrians, were utilized in the case of 90 degrees, those characteristics of wider ranges were induced in the case of 60 degrees, which led to diffused characteristics, which is judged by us to increase the probability of false recognition. Recognition conduction time was analyzed as approximately $20 \mathrm{~ms}$ in the suggested method for one image whose characteristics has been extracted, and in the other methods the time was analyzed similarly.

\section{Conclusion}

A new system for detecting two-wheelers was suggested by applying the saving method of new characteristic vectors. Ordinary HOG has two kinds of spatial regions: small (cells) and large (blocks). And it is based on overlapping and dense encoding of image regions. This paper suggests that feature vectors were saved by each spatial region periodicity (for cell and block) similar to scattering, though calculation for feature extraction used dens and overlapping. Characteristic vectors were obtained, saved by collecting local cells once, those of which were normalized and collected once again, and then finally the characteristic vectors were extracted. The Adaboost Method was applied for a speedy classification of two-wheelers; the results compared with existing methods have been shown that it is a highly improved system performance. When applied in an actual system, it has a much higher accuracy than the other ordinary existing methods, as well. The characteristics that can be better classified in cases similar to two-wheelers, as demonstrated in the experiments, will be developed and the bicycles with different attitudes and shapes will be analyzed more precisely in future studies.

\section{References}

[1] H. Jung, Y. Ehara, J. K. Tan, H. Kim, and S. Ishikawa, "Applying MSC-HOG Feature to the Detection of Human on a Bicycle", International Conference on Control, (2012) October 17-21; Jeju Island, Korea

[2] H. Cho, P. E. Rybski, and W. Zhang, "Vision-based Bicyclist Detection and Tracking for Intelligent Vehicles", IEEE Intelligent Vehicle Symposium, (2010) June 21-24; Univ. of California

[3] Y. Xu, X. Cao, and H. Qiao, "An Efficient Tree Classifier Ensemble-based Approach for Pedestrian Detection", IEEE Transaction on System, Man and Cybernetics, vol. 41, no. 1, (2011).

[4] X. Liu and K. Fujimura, "Pedestrian detection using stereo night vision", IEEE Vehicle Technology, vol. 53 , no. 6, (2004).

[5] M. Bertozzi, A. Broggi, A. Lasagni and M. D. Rose, "Infrared stereo vision-based pedestrian detection", IEEE Intell. Veh. Symp., (2005) June 6-8.

[6] S. Sato, M. Hashimoto, M. Takita, K. Takagi and T. Ogawa, "Multilayer lidar-based pedestrian tracking in urban environments", IEEE Intelligent Vehicles Symposium, (2010) June 21-24 June; San Diego, USA.

[7] H. G. Jung, Y. H. Lee, P. J. Yoon, I. Y. Hwang and J. Kim, "Sensor fusion based obstacle detection/classification for active pedestrian protection system", Edited, G. Bebis, Advance in Vision Computing, Springer Berlin Heidelberg, vol. 4292, (2006), pp. 294-305.

[8] T. Gandhi and M. M. Trivedi, "Pedestrian Protection Systems: Issues, Survey, and Challenges", IEEE Transaction on Intelligent Transportation Systems, vol. 8, no. 3, (2007).

[9] M. Enzweiler and D. M. Gavrila, "Monocular Pedestrian Detection: Survey and Experiments", IEEE Tansaction Pattern Analysis and Machine Intelligent, vol. 31, no. 12, (2008).

[10] I. P. Alonso, D. F. Llorca, M. A. Sotelo, L. M. Bergasa, P. R. de Toro, J. Nuevo, M. Ocana and A. G. Garrido, "Combination of feature extraction methods for SVM pedestrian detection", IEEE Transaction Pattern Analysis and Machine Intelligent, vol. 8, no. 2, (2007).

[11] H. G. Jung, "Pedestrian Protection System: Review and Problems", KSAE 2010 Annual Conference, (2010) November 14-16; Daegu, Korea.

[12] O. Tuzel, F. Porikli and P. Meer, "Human detection via classification on Riemannian manifolds", Proceeding of Computer Vision and Pattern Recognition, (2007) June 17-22; Minneapolis, USA. 
[13] L. Yu, F. Zhao and Z. An, "Locally Assembled Binary Feature with Feed-forward Cascade for pedestrian Detection in Intelligent Vehicles.”, Proceeding $9^{\text {th }}$ Conference on Cognitive Informatics, (2010) July 79; Beijing, China.

[14] L. Oliveria, P. Peixoto and U. Nunes, "A Hierarchical Fuzzy Integration of Local and Global Featurebased Classfiers to Recognize Objects in Autonomous Vehicles", ICRA 2007 Workshop: Planning, Perception and Navigation for Intelligent Vehicles, (2007) April 10-14; Rome, Italy.

[15] L Oliveira, A. L. Koerich, M. Mansano, A. S. Britto, "2D Principal Component Analysis for Face and Facial-Expression Recognition”, Computing in Science \& Engineering, vol.13, no. 3, (2011).

[16] P. F. Felzenzwalb, R. B. Girshick, D. Mcallester and D. Ramanan, "Object Detection with Discriminatively Trained Part-Based Models", IEEE Transaction Pattern Analysis and machine Intelligen, vol. 32, no. 9, (2010).

[17] D. M. Gavrila and S. Munder, "Multi-cue pedestrian detection and tracking from a moving vehicle", International Journal of Computer Vision, vol. 73, no. 1, (2007).

[18] P. Sabzmeydani and G. Mori, "Detecting Pedestrians by learning shapelet feature", Proceeding of Computer Vision and Pattern Recognition, (2007) June 17-22; Minneapolis, USA.

[19] T. Ahonen, A. Hadid and M. Pietikainen, "Face description with local binary patters: Application to face recognition”, IEEE Transaction Pattern Analysis and machine Intelligent, vol. 28, no. 12, (2006).

[20] N. Dalal and B. Triggs, "Histogram of Oriented Gradients for Human Detection", IEEE Computer. Vision Pattern Recognition (2005) June 25; San Diego, USA.

[21] S. Pavani, D. Delgado and A. F. Frangi, "Haar-like features with optionally weighted rectangles for rapid object detection", Pattern Recognitio, vol. 43, no. 1, (2010).

[22] C. Papageorgiou and T. Poggio, "A trainable system for object detection", International Journal of Computer Vision, vol. 38, no. 1, (2000).

[23] X. Y. Wang, T. X. Han and S. Yan, "An HOG-LBP human detector with partial occlusion handling”, International Conference on Computer Vision, (2009) September 29 -October 2; Kyoto, Japan.

[24] S. H. Lee, Y. H. Lee, T. S. Kim and H, D. Seo, "Pedestrian Recognition using the Cascade Method of Independent Features based on Adaboost Algorithm", The Journal of KIIT, vol. 8, no. 8, (2010).

[25] X. B. Cao, Y. W. Xu, D. Chen, and H. Qiao, "Associated evolution of a support vector machine-based classifier for pedestrian detection", Information Sciences, vol. 179, no. 8, (2009).

[26] D. Gerónimo, A. M. López, A. D. Sappa and T. Graf, "Survey of Pedestrian Dectection for Advanced Driver Assistance Systems", IEEE Transaction Pattern Analysis and Machine Intelligent, vol. 32, no. 7, (2010).

[27] Q. Zhu, M. C. Yeh, K. T. Cheng and S. Avidan, "Fast human detection using a cascade of histograms of oriented gradients", IEEE Conference on Computer Vision and Pattern Recognition, (2006) June 17-22; New York, USA

[28] T. Watanabe, S. Ito and K. Yokoi, "Co-occurrence Histogram of Oriented Gradients for Detection Advances in Image and Video Technology' Lecture Notes in Computer Science, vol. 5414, (2009).

[29] P. Viola, M. Jones, and M. Snow, "Detection pedestrians using patterns of motion and appearance", 9th IEEE International Conference on Computer Vision, (2003) October 13-16; Nice, France.

[30] http://en.wikipedia.org/wiki/Receiver_operating_characteristic

\section{Authors}
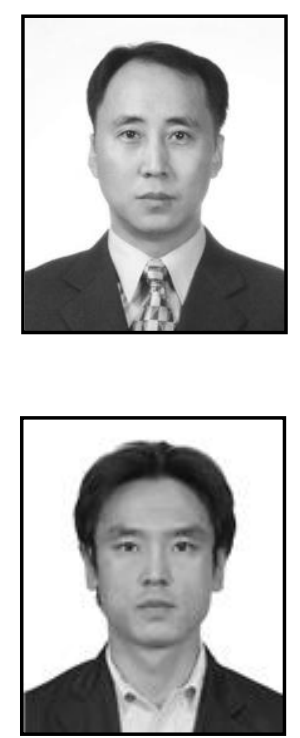

Yeunghak Lee, he received his Ph.D. degree from Yeungnam University, Korea, in 2003. He had one year experience at University of Cardiff as postdoc research fellow. Currently, he is a professor of department of avionic electronic engineering at Kyungwoon University. And he is contributing himself as a management editor for the Journal of Multimedia and Information Systems. His research interests include pattern recognition, embedded system and computer vision.

Sanghoon Lee, he received his PH.D degree from Yeungnam University, Korea, in 2011. He had four years experience at Daekyeong Institute for Regional Program Evaluation. Currently, he is a director of department of Gyeongbuk Science \& Technology Promotion Center at Gumi Electronics \& Information Technology Research Institute. His research interests include rehabilitation engineering, image processing and embedded system. 

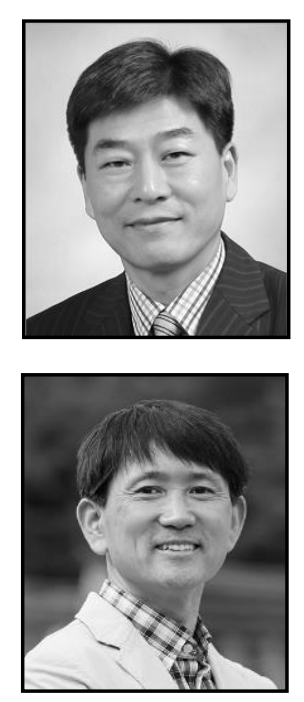

Taesun Kim, he is a professor of department of avionic electronic engineering at Kyungwoon University. He holds a doctorate degree in Electronic Engineering from Yeungnam University. From 1991 to 1995, he was a researcher in the TV Research Institute at LG Electronics. His research interests include image analysis, image system and signal processing.

Jaechang Shim, he is a Professor in the Department of Computer Engineering at Andong National University. His research interests include pattern recognition, image processing, embedded vision system, biometric authentication, and 3D object recognition. $\mathrm{He}$ received the best paper awards from IAPR in 1998. He is also a cofounder of PAMI Inc. since 1998. 